\title{
Europäische Besitzungen. Zur gespaltenen Possession im europäischen Sprachvergleich ${ }^{1}$
}

\begin{abstract}
Die Aufbauprinzipien europäischer Possessionssysteme basieren viel öfter als gemeinhin angenommen auf gespaltener Possession. Dieses im Deutschen nicht prominente Phänomen wird anhand von Daten aus ausgewählten Beispielsprachen als in Europa durchaus etablierte Erscheinung vorgestellt. Die mit ihm verbundenen semantischen und morphosyntaktischen Aspekte werden anhand von qualitativen Korpusdaten diskutiert. Die Restriktionen, denen sie unterliegen, sind ebenfalls Gegenstand der Diskussion. In den Schlussfolgerungen wird dafür plädiert, künftig der Possession bei vergleichenden Studien zu europäischen Sprachen mehr Beachtung zu schenken.
\end{abstract}

\section{Zum Einstieg}

Den Gegenstand meiner Betrachtungen bildet die gespaltene Possession (ab hier kurz: GP), die ich an Hand einer kleinen Auswahl von Fällen aus Europa illustriere und auswerte. Vergleiche zum Deutschen werden an geeigneter Stelle gezogen, dessen Eigenschaften ansonsten als bekannt vorausgesetzt werden. Die Grundbegrifflichkeit der GP ist durch einen knappen Beitrag von Dahl/Koptjevskaja-Tamm (1998) motiviert, der von ,alienability splits“ handelt. Die Erforschung dieser und anderer Formen der GP ist nicht zuletzt von großem Interesse für die lexikalische oder semantische Typologie (Evans 2011). Meine Studie fällt in die Kategorie der Possessionsforschung, die seit Seiler (1973) einen enormen Aufschwung genommen hat und heute dank einflussreicher Arbeiten zum Beispiel von Heine (1997) und jetzt auch Stassen (2009) aus der funktional-typologisch ausgerichteten Sprachwissenschaft nicht mehr weg zu denken ist. Als eigenständiges Thema erfährt GP in der Possessionsforschung allerdings erst seit jüngerer Zeit angemessene Beachtung (Stolz et al. 2008), wobei noch viel empirische Kernarbeit in zu-

\footnotetext{
Diese Studie gehört in das Nachfeld des Projektes „Alienabilität/Inalienabilität in Europa“, das 2002-2004 von der Deutschen Forschungsgemeinschaft an der Universität Bremen gefördert wurde. Ich danke den Mitgliedern des Nachwuchsnetzwerkes und Arbeitskreises „Sprachkontakt und Sprachvergleich“ am selben Ort für ihre Unterstützung sowie meinen Diskutanten auf der in diesem Band dokumentierten IDS-Jahrestagung für ihre anregenden Hinweise. Lutz Gunkel und Gisela Zifonun gebührt herzlicher Dank für die kritische Durchsicht der ersten Version meines Beitrags. Maris Camilleri, Ray Fabri und Tamar Reseck haben mir wichtige Antworten auf Fragen zu ihren Muttersprachen gegeben. Für Form und Inhalt dieses Beitrages trage ich dessen ungeachtet die alleinige Verantwortung.
} 
künftigen Projekten zu leisten sein wird. Mit diesem Beitrag möchte ich wenigstens andeuten, dass sich in diesem Sinne das vertiefte Studium europäischer Possessionssysteme lohnt.

Die aufgrund ihrer Dominanz in der einschlägigen Literatur (Chappell/ McGregor 1996) am besten bekannte Erscheinungsform von GP ist die formale Distinktion von veräußerlichem und unveräußerlichem Besitz (Nichols 1988), die üblicherweise und weit überwiegend mit Beispielen aus außereuropäischen Sprachen belegt wird (Seiler 1983, S. 20-21). Stellvertretend für diese Praxis steht das Beispielpaar unter (1) aus dem Zapotekischen (Butler 1988, S. 192, 195).

\section{Zapotekisch}

(a) inalienabel: yichj- „Kopf“ $\rightarrow$ yichj-a' ,,mein Kopf“"

(b) alienabel: bey "Tuch“ $\rightarrow$ x-pey-a', ,mein Tuch“

Im Zapotekischen tragen Körperteilbezeichnungen und Verwandtschaftstermini Suffixe wie das $-a$ ' in (1a), die angeben, welche Person als Possessor - hier die 1. Person Singular - fungiert. Substantive, die anderen semantischen Klassen angehören, müssen für die Zwecke der Possession zusätzlich zu den Possessorsuffixen noch ein (die Desonorisierung des Anlautkonsonanten bewirkendes) Präfix $x$ - tragen, das sie als possediert ausweist, was in (1b) der Fall ist. D.h. also, dass die Elemente einer Wortart (Nomen) für den Ausdruck derselben Kategorie (Possession) auf unterschiedliche Konstruktionen ((1a) und (1b)) zurückgreifen. Die Wahl der Konstruktionen korreliert mit semantischen Unterschieden der Substantive, die das Possessum repräsentieren. Die formalen Regelungen sind obligatorisch und overt; an ihnen sind funktional spezialisierte Marker beteiligt. Es gibt, mit anderen Worten, eine semantisch motivierte Spaltung auf morphosyntaktischer Ebene.

Die Alienabilitätskorrelation in außereuropäischen Sprachen erschöpft die Phänomenologie der GP jedoch längst nicht. Auch im Deutschen finden sich Belege für GP, wenn man die Verteilung der für possessivische Zwecke genutzten Konstruktionen unter die Lupe nimmt. Unter (2) sind die Möglichkeiten verzeichnet, wie standardsprachlich mit NP-interner Possession verfahren werden kann, wenn der Possessor durch einen Eigennamen bzw. durch ein Appelativum vertreten ist. ${ }^{2}$

(2) Deutsch

\begin{tabular}{|c|c|c|c|c|c|c|c|c|c|}
\hline \multicolumn{4}{|c|}{ Eigenname } & \multicolumn{6}{|c|}{ Appellativum } \\
\hline \multirow{2}{*}{$\begin{array}{l}\text { die } \\
\text { die }\end{array}$} & \multicolumn{2}{|c|}{ Hütte } & Hagrids & \multirow{2}{*}{\begin{tabular}{|l} 
die \\
die \\
\end{tabular}} & \multicolumn{2}{|c|}{ Hütte } & \multicolumn{2}{|l|}{ des } & Riesen \\
\hline & Hütte & von & Hagrid & & Hütte & von & & dem & Riesen \\
\hline \multicolumn{2}{|c|}{ Hagrids } & \multicolumn{2}{|c|}{ Hütte } & \multicolumn{2}{|l|}{ des } & \multicolumn{2}{|c|}{ Riesen } & \multicolumn{2}{|c|}{ Hütte } \\
\hline
\end{tabular}

Für die nicht-standardsprachliche Konstruktion Hagrid seine Hütte siehe Zifonun (2003). 
Die unterschiedliche Grautönung der Zellen auf der Seite des Appellativums deutet den abnehmenden Akzeptabilitätsgrad der verzeichneten Konstruktionen an. Nur bei Eigennamen ist es in stilistisch neutraler Form möglich, den genitivisch flektierten Possessor dem Ausdruck des Possessums voranzustellen. ${ }^{3}$ Appellativa, die in dieser Position verwendet werden, sind nur dann zulässig, wenn bewusst der anachronistische Effekt der Konstruktion genutzt werden soll. In diesem Fall sind es also spezifische Eigenschaften des Possessors, die zur GP beitragen: Eigennamen verhalten sich morphosyntaktisch anders als Appellativa, wenn sie die Rolle von Possessoren spielen. Im Gegensatz zum zapotekischen Beispiel ist die GP im Deutschen weniger leicht zu erkennen, da sie mit weitgehend fakultativen Stellungsvarianten verknüpft ist, ohne dass ein identifizierbares spezialisiertes Morphem die Konstruktionen voneinander unterscheidet.

Für viele Sprachen Europas ist die Beleglage ähnlich gelagert, sodass die Instanzen von GP zu wenig prominent erscheinen, um in crosslinguistisch ausgerichteten Untersuchungen berücksichtigt zu werden. Die europäische grammatikografische Tradition sieht keinen systematischen Ort für Possession, geschweige denn für GP vor, sodass entsprechende Evidenz in den herkömmlichen deskriptiven Materialien nur schwer zu finden ist. Zudem manifestieren sich viele Fälle von europäischer GP in eher subtilen Restriktionen über die Kombination bestimmter meistens freier Morpheme und/oder über die Linearisierungsmöglichkeiten innerhalb einer possessivischen Konstruktion. Häufig sind Morpheme entscheidend, die außerhalb des possessivischen Kontextes ganz andere Funktionen ausüben (wie etwa der definite Artikel u.a. in verschiedenen romanischen Sprachen (Spanoghe 1995) oder der Relativsatz im Maltesischen (Stolz im Druck)). Die sich ergebenden Distinktionen unterliegen darüber hinaus unter bestimmten (z.T. recht häufig gegebenen) Bedingungen der Neutralisierung. Europäische GP-Fälle sind nicht selten mit dem Einsatz von sekundären Kodierungsstrategien verbunden, die in vielen ausschließlich auf primäre Ausdrucksmittel fixierten Ansätzen automatisch ausgeschlossen werden (Stassen 2009, S. 25-34, 45-47). Es nimmt daher nicht Wunder, dass Nichols (1992, S. 123, 294) im globalen Vergleich Europa als eine weltweit ziemlich isolierte Zone versteht, in der die Alienabilitätskorrelation keine grammatisch relevante Rolle spielt. Haspelmath (2001) nimmt diesen Faden auf und präzisiert die

Ich kann hier der Frage nicht weiter nachgehen, ob Konstruktionen mit nachgestelltem Eigennamen grundsätzlich akzeptabler sind als solche mit vorangestelltem Appellativum. Lutz Gunkel (persönliche Mitteilung), den ich hier direkt zitiere, macht mich darauf aufmerksam, dass zunächst zwischen personalen und geografischen Eigennamen zu unterscheiden ist. Bei letzteren steht der Nachstellung nichts im Wege, vgl. die Eroberung Roms, die Unabbängigkeit Osttimors. Bei personalen Eigennamen gibt es Beschränkungen, wobei die Länge des Namens ein Faktor ist. Demnach sind kurze Eigennamen „,weniger üblich“ als lange, also eine bekannte Fuge Bachs vs. eine bekannte Fuge Johann Sebastian Bachs. Wahrscheinlich hängt die Akzeptabilität der Nachstellung zusätzlich vom Register ab: bei hohem eher akzeptabel, sonst weniger. 
Hypothese dahingehend, dass er das Fehlen der Alienabilitätskorrelation als eine der gemeinsamen negativen Eigenschaften der SAE-Sprachen ansieht.

Die Sprachen Europas kommen in der Possessionsforschung keineswegs zu kurz. Auch Arbeiten, in denen beispielsweise über den Ausdruck von inalienabler/alienabler Possession meistens in modernen Einzelsprachen (z.B. Hentschel/Menzel 2002 zum Russischen) gehandelt wird, sind in ansehnlicher Zahl erschienen. Thematisch besonders beliebt ist die Possessoranhebung, die König/Haspelmath (1997) als SAE-Merkmal charakterisieren, wobei es deutlich hervortretende Zusammenhänge zwischen Inalienabilität und Possessoranhebung zu geben scheint. Mit klarem Bezug auf inalienable Possession befasst sich Manzelli (2007) mit der pronominalen Possession. In areal-typologischer paneuropäischer Perspektive betrachtet Koptjevskaja-Tamm (2002 und anderen Arbeiten) die Grammatik der adnominalen Possession und identifiziert dabei punktuell Bezüge zur Alienabilitätskorrelation. Diese und andere Hinweise auf mögliche Fälle von GP in den Sprachen Europas sind lange Zeit isoliert geblieben und haben keinen Einfluss auf die breite linguistische „Meinungsbildung“ gehabt.

Das Vorurteil, in Europa sei die Alienabilitätskorrelation grammatisch grundsätzlich irrelevant, wird allerdings durch Koptjevskaja-Tamm (1996) zum Maltesischen und in den von mir mit verantworteten Schriften zum Färöischen (Stolz/Gorsemann 2001), Isländischen (Stolz 2004) und zur europaweiten Ausgestaltung von GP (Stolz et al. 2008) auch aus typologischer Sicht widerlegt. Auf diesen Vorarbeiten aufbauend gehe ich dieses Mal verstärkt auf die Faktoren ein, die gleich in mehreren europäischen Sprachen GP bewirken bzw. einschränken. Für diese Zwecke gebe ich in Abschnitt 2 eine Reihe von Vorklärungen. Der empirische Hauptteil 3 ist intern nach den GP-auslösenden bzw. -unterbindenden Faktoren gegliedert. Abschnitt 4 ist als Schlusskapitel der Beurteilung des Gesamtbefundes gewidmet. Diskutiert werden ausschließlich besonders augenfällige Phänomene ohne Anspruch auf Vollständigkeit.

Hinsichtlich der theoretischen Grundlagen steht diese Arbeit der Basic Linguistic Theory (Dixon 2010) nahe und verortet sich im näheren Umfeld des Funktionalismus (Givón 2001, S. 1-41). Es müssen darüber hinaus Anleihen bei der Konstruktionsgrammatik (Stefanowitsch 2006) gemacht werden. Methodologisch betreibe ich korpusgestützten typologischen Sprachvergleich (Cysouw/Wälchli 2007), der unter Berücksichtigung der üblichen deskriptiv-linguistischen Materialien ergänzt wird. Dabei gehe ich ausschließlich qualitativ vor. Es wird überwiegend auf das literarische Parallelkorpus von Übersetzungen des ersten Bandes der Harry-Potter-Heptalogie zurückgegriffen (Stolz 2007). Die Daten reflektieren daher den standardnahen Sprachgebrauch vornehmlich im schriftlichen Register der Beispielsprachen. Die Blickrichtung ist ausschließlich synchron. Bei der Beschreibung posses- 
sivischer Konstruktionen folge ich terminologisch Heine (1997). Die diesmal herangezogenen Sprachen (Albanisch (Buchholz/Fiedler 1987, S. 284 291), Färöisch (Thráinsson et al. 2004, S. 62-65), Georgisch (Fähnrich 1986), Isländisch (Thráinsson 2010, S. 92-96), Italienisch (Renzi/Salvi/Cardinaletti (Hg.) 1988), Maltesisch (Borg/Azzopardi-Alexander 1997, S. 111-113), Portugiesisch (Cunha/Cintra 1985)) bilden eine sehr kleine Teilmenge des Samples von Stolz et al. (2008). Den hier zugrunde gelegten und sehr weit gefassten Europabegriff erläutern Stolz/Stroh/Urdze (2003).

\section{Vorklärungen}

Possession setzt eine binäre Relation a $\mathfrak{R} b$ voraus, die den possessivischen Situationen zu Grunde liegt, in denen zwei Partizipanten auftreten, wobei der Possessor über das Possessum Kontrolle ausübt. Schematisch ergeben sich mit der Relation dann drei Variablen als Kernkomponenten einer possessivischen Situation. Theoretisch bietet jede der Variablen die Möglichkeit, GP auszulösen, je nachdem welcher semantischen oder anderen Klasse die Füller angehören, die in einer Sachverhaltsbeschreibung die Partizipantenrollen ausüben bzw. die possessivische Relation spezifizieren. Es lassen sich für jede der drei Positionen zwei Parameter identifizieren, nach denen Variation stattfinden kann (Heine 1997, S. 33-40). Die Tabelle unter (3) stellt dies schematisch dar.

(3) Parameter der Variationsmöglichkeiten

\begin{tabular}{|l|l|l|}
\hline Possessor & Relation & Possessum \\
\hline$[ \pm$ menschlich $]$ & {$[ \pm$ permanent $]$} & {$[ \pm$ alienabel $]$} \\
\hline$[ \pm$ belebt $]$ & {$[ \pm$ physisch $]$} & {$[ \pm$ konkret $]$} \\
\hline
\end{tabular}

Sowohl Heine (1997, S. 39-40) als auch Stassen (2009, S. 35) gehen aus guten arbeitstechnischen Gründen von Prototypen aus, bei denen der Possessor als [+menschlich] und die Ausprägung der Relation als [+ permanent] invariabel bleiben und nur beim Possessum semantische Variation zugelassen wird, sodass der Horizont von vornherein auf GP-Fälle vom Schlage der Alienabilitätskorrelation beschränkt wird. Verstärkt wird diese Einschränkung noch durch die Festlegung auf indefinite unbelebte Possessa mit der Eigenschaft [+konkret] sowie den Ausschluss der gehören-Konstruktionen (Seiler 1983, S. 61) zu Gunsten der baben-Konstruktionen (Heine 1997, S. 29 33). In der Praxis wird außerdem possessivischen Konstruktionen der Vorzug gegeben, in denen die beteiligten NPn keine Attribute enthalten, ein eventuelles Possessionsverb nicht weiter adverbial modifiziert wird und positive Polarität vorliegt. Kurz gesagt werden alle Parameter hinsichtlich der Markiertheitswerte auf null gesetzt. 
Trotz der erheblichen methodologischen Bedenken von Stassen (2009, S. 38-47) weiche ich in diesem Beitrag verschiedentlich von dem oben skizzierten Prototyp ab, da GP-Fälle in europäischen Sprachen nicht zuletzt auch dort auftreten, wo entweder die Grenze zur marginalen Possession überschritten wird oder andere Abweichungen vom Prototyp bedeutsam werden. Dies unterstützt die Ansicht, dass für ein Gesamtverständnis der Grammatik von Possessionssystemen über den Prototypenansatz hinausgegangen werden muss (Stolz et al. 2008, S. 21-24), was meine Studie nur in bescheidenem Umfang leistet. Eine Umorientierung der Possessionsforschung auf den kanonischen Ansatz in der Typologie (Corbett 2005) könnte zukünftig zum Erkenntnisgewinn beitragen.

Unter (4) fasse ich schematisch die grundlegenden morphosyntaktischen Muster zusammen, die für die Einteilung possessivischer Konstruktionen wichtig sind. ${ }^{4}$

(4) Synopse der possessivischen Morphosyntax

\begin{tabular}{|l|l|l|l|l|l|}
\hline Ebene & Possession & Possessor & Relation & Possessum & Typisches \\
\hline \multirow{2}{*}{$\mathrm{NP}$} & adnominal & $\mathrm{NP}_{[\text {Modifikato] }]}$ & $\mathrm{X}$ & $\mathrm{NP}_{[\text {Kopf] }}$ & $\begin{array}{l}\text { Kasus („Genitiv } \\
\sim \text { PP } \sim \text { Juxtaposition }\end{array}$ \\
\cline { 2 - 6 } & pronominal & Pro $_{[\text {Modifikator] }}$ & $\mathrm{X}$ & $\mathrm{NP}_{[\text {Kopf] }}$ & $\begin{array}{l}\text { Possessivpronomen } \\
\sim \text { PP }\end{array}$ \\
\hline (S) VP & prädikativ & $\mathrm{NP} /$ Pro & $\mathrm{V}_{[\text {Kopf] }}$ & $\mathrm{NP}_{[\text {Komplement] }}$ & haben-Verb \\
\hline
\end{tabular}

Das Element X kann gegebenenfalls phonologisch unrealisiert bleiben, was in Sprachen, die nicht dem Action-Schema (Heine 1997, S. 47-50) folgen, tempusabhängig auch bei $\mathrm{V}$ in der prädikativen Possession der Fall sein kann, wenn beispielsweise die Kopula im Präsens phonologisch nicht realisiert wird (Heine 1997, S. 51). Den Rahmen für die Vergleichbarkeit der in Abschnitt 3 präsentierten Fakten bildet das deutsche Possessionssystem, in dem die Domäne von pronominaler, adnominaler und prädikativer Possession durch die Verwendbarkeit pro syntaktische Ebene jeweils gleicher Konstruktionen sehr weit geschnitten ist. Als Faustregel gilt, dass alles das unter Possession fällt, was im Deutschen eine übersetzungsäquivalente Konstruktion hat, in der das Possessivpronomen, ${ }^{5}$ der adnominale Genitiv oder seine Ersatzformen oder das Possessionsverb auftreten.

$4 \quad$ Ich behalte die terminologische Unterscheidung zwischen adnominaler und pronominaler Possession bei, die in der anglophonen Literatur üblich geworden ist, obwohl Lutz Gunkel (persönliche Mitteilung) zuzustimmen ist, dass sie unglücklich ist, denn die pronominalen Possessiva fungieren ja auch adnominal.

5 Gisela Zifonun (persönliche Mitteilung) macht mich darauf aufmerksam, dass das Possessivpronomen im Deutschen das Possessum definit macht. Inwiefern wir diesen Umstand bereits als Abweichung vom Prototyp verstehen können, muss an anderer Stelle diskutiert werden. 


\section{GP und ihre Verursacher}

In diesem empirischen Abschnitt mustere ich schrittweise die Faktoren durch, für die es jeweils mindestens zwei Sprachen gibt, in denen aufgrund des Faktors GP auftritt. Den Auftakt machen GP-Fälle, die sich in die bekannten Muster der Alienabilitätskorrelation einreihen (= Abschnitt 3.1). Von dort ausgehend weite ich die Betrachtung auf andere Bereiche der GP aus (= Abschnitt 3.2) und komme in Abschnitt 3.3 auf Störfaktoren zu sprechen, die zur Neutralisierung von possessivischen Distinktionen beitragen.

\subsection{Inalienabilität}

Heine (1997, S. 10) präsentiert eine hierarchisch geordnete Liste von konzeptuellen Bereichen, die in Sprachen mit formaler Alienabilitätskorrelation unter die Rubrik der inalienablen Konzepte fallen. Diese Liste ist unter (5) in leicht angepasster Form wiedergegeben.

(5) Hierarchie der inalienablen Konzepte

\begin{tabular}{|c|l|l|}
\hline & Konzeptuelle Domäne & potenzielle Fälle \\
\hline 1 & Verwandtschaftsverhältnisse & Mutter, Sohn usw. \\
\hline 2 & Körperteile & Kopf, Rücken usw. \\
\hline 3 & Relationale Konzepte & Oberseite, Innenseite usw. \\
\hline 4 & Teil-Ganzes-Beziehungen & Ast (eines Baumes), Dach (eines Hauses) usw. \\
\hline 5 & Physisch-mentale Zustände & Angst, Hunger usw. \\
\hline 6 & Nominalisierungen & (sein) Singen, (ihr) Schnarchen usw. \\
\hline 7 & individuelle Konzepte & Name, Geruch usw. \\
\hline
\end{tabular}

Sprachvergleichend ergibt sich, dass die auf den Positionen 1-2 stehenden Konzeptbereiche den Kern der Inalienabilität ausmachen, ohne dass sich Verwandtschaftsverhältnisse und Körperteile untereinander noch in eine eindeutige Reihenfolge bringen ließen (Heine 1997, S. 11-12): Mindestens eine der beiden konzeptuellen Domänen ist auf jeden Fall an den Alienabilitätskorrelationen der Sprachen der Welt beteiligt - und genauso ist es auch in Europa.

\subsubsection{Verwandtschaftsverhältnisse}

Dahl/Koptjevskaja-Tamm (2001, S. 208-211) gehen in ihrem typologischen Überblick über die grammatischen Eigenschaften von Verwandtschaftstermini kursorisch auch auf ihre Rolle in Possessionssystemen ein. Es lohnt sich allerdings, diesem Aspekt noch weitaus mehr Aufmerksamkeit zu schenken. 
In mehreren Sprachen Europas verhalten sich alle oder bestimmte Verwandtschaftstermini morphosyntaktisch anders als sonstige Nomina, wenn sie possediert sind. So stehen Sprechern der afroasiatischen Sprache Maltesisch zwei possessivische Konstruktionen auf der NP-Ebene zur Verfügung (Stolz et al. 2008, S. 44-94). Ist das Possessum ein alienables Konzept, wird der Possessor als präpositionales Attribut ausgedrückt. Dies ist in (6a) ${ }^{6}$ mit den Possessa curkett „Ring“ und djamant „Diamant“ der Fall, die durch die (pronominal flektierte) Präposition $t a$ ',,von“ mit dem Possessor verbunden sind. Ist das Possessum hingegen ein Verwandtschaftskonzept, erfolgt bei pronominaler Possession die Suffigierung eines Possessorzeichens am possedierten Nomen wie bei missier-u, sein Vater" in (6a), während bei adnominaler Possession die Juxtaposition (genannt status constructus) zur Anwendung kommt, was durch omm l-ghasafar ,die Mutter der Vögel““ in (6b) belegt ist.

(6) Maltesisch

(6a) [Gahan 110]

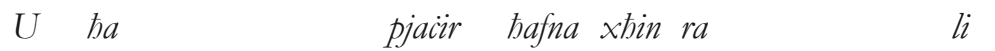

und nehm(3SG.M.PERF) Freude viel als seh(3SG.M.PERF) dass

[i-iurkett ta' [missier-u]] u [d-djamant tieghu]

DET-Ring von Vater-3SG.M und DET-Diamant von:3SG.M kienet tilbishom ukoll.

sei:3SG.F.PERF 3SG.F.IMPERF:anzieh:3Pl auch

„Und er freute sich sehr, als er sah, dass sie auch den Ring seines Vaters und seinen Diamanten trug.“

(6b) [Gahan 69]: status constructus

[Omm l-ghasafar] waslet $u$ kienet

Mutter DET-Vogel:PL ankomm:3SG.F.PERF und sei:3SG.F.PERF kbira நafna.

groß:F viel

„Die Mutter der Vögel kam an und war sehr groß.“

Im südlichen und südöstlichen Europa bieten Italienisch, Albanisch und Georgisch ebenfalls Evidenz dafür, dass Verwandtschaftsverhältnisse im Rahmen der Possession gewissermaßen „Sonderrechte“ genießen (Stolz et al. 2008, S. 318-335). Im Unterschied zum Maltesischen ist dies nur in der pronominalen Possession gegeben und außerdem ist jedes Mal nur eine (wenn

6 Bei der Beispielangabe stehen die possessivischen Konstruktionen in der objektsprachlichen Zeile in eckigen Klammern. Morphemtrennstriche verwende ich sparsam. Die in der Glossierung verwendeten Kürzel buchstabiere ich am Textende aus. Die Primärquellen, denen die Belege entnommen sind, werden nach der Beispielnummer in eckigen Klammern per Sigel und Seitenzahl identifiziert. Soweit nicht anders vermerkt stammen alle Übersetzungen von mir. 
auch verschieden große) Untergruppe der Verwandtschaftsverhältnisse in diesem Sinne privilegiert. Dass GP vorliegt, lässt sich an den Versionen zweier Sätze aus dem Parallelkorpus belegen. Während im Deutschen der pronominale Possessor bei den Possessa Name und Vater in (7a-b) gleich ist, verwenden Georgisch in (8a-b), Albanisch in (9a-b) und Italienisch in $(10 a-b)$ jeweils zwei verschiedene Konstruktionen.

(7) Deutsch [HP Deutsch 120]

(7a) [-Verwandtschaft] Meinst wobl, [mein Name] ist komisch, was?

(7b) [+Verwandtschaft $]$ [Mein Vater] hat mir gesagt ...

(8) Georgisch [HP Georgisch 101]

(8a) Possessum [-Verwandtschaft]

$\mathrm{R} a, \quad[$ čem-s saxel-ze] gec'ineba?

was mein-DAT Name-auf 2SG:CV:lach:3SG

„Was, du lachst über meinen Namen?“

(8b) Possessum [+Verwandtschaft]

[Mama-čemi] ambobs...

Vater-mein:NOM sag:3SG

„Mein Vater sagt ...“

(9) Albanisch [HP Albanisch 92-93]

(9a) Possessum [-Verwandtschaft]

$T \ddot{e}$ bën për të qeshur [mbiemr-i im]?

dich mach(3SG) für INF gelacht Nachname-DET.M.SG mein „Macht dich mein Nachname lachen?““

(9b) Possessum [+Verwandtschaft]

[Im atë] mё ka thënё se ...

mein Vater mir hab(3SG) gesagt dass

„Mein Vater hat mir gesagt ..."

(10) Italienisch [HP Italienisch 106]

(10a) Possessum [-Verwandtschaft]

Trovi buffo [il mio nome], vero?

find:2SG komisch DET mein Name wahr

„Du findest meinen Namen komisch, nicht wahr?“

(10b) Possessum [+Verwandtschaft]

[Mio padre] mi ha detto che...

mein Vater mir hab(3SG) gesagt dass

„Mein Vater hat mir gesagt, dass ..." 
Im Georgischen können nur Termini für Verwandtschaft mit älteren Generationen Possessorsuffixe tragen (wie mama-čemi „,mein Vater"), wohingegen alle anderen Possessa pränominale freie Possessivpronomina verlangen (wie čmi saxeli „mein Name“) (Dahl/Koptjevskaja-Tamm 1998). Im Albanischen wird zwischen zwei Schichten von Verwandtschaftsbezeichnungen unterschieden. Die so genannten alten Verwandtschaftsbezeichnungen nehmen pränominale Possessivpronomina (wie im atë „,mein Vater"), während ,neue“ Verwandtschaftsbezeichnungen sich so wie alle übrigen Appellativa verhalten, indem sie postnominale Possessiva verlangen (wie mbiemri im ,mein Nachname"). Die Gegebenheiten im Italienischen sehen vor, dass bei pronominaler Possession der definite Artikel das Possessivpronomen begleiten muss (wie il mio nome „mein Name“), während der Einsatz des definiten Artikels bei possedierten Verwandtschaftstermini blockiert ist (wie mio padre „mein Vater"), was Haspelmath (1999) mit frequenz-basierten sprachökonomischen Erwägungen zu begründen sucht.

Das ist aber längst nicht die ganze Geschichte. Italienisch, Albanisch und Georgisch teilen noch die Eigenschaft miteinander, dass die oben skizzierten Regeln nur für einen Ausschnitt des Possessorparadigmas gelten, sodass dieses seinerseits der GP unterliegt. Unter (11) geben die grauschattierten Zellen an, wo die grammatischen Privilegien der Verwandtschaftstermini aufgehoben werden. Die Kombination Y/Z besagt, dass zwei gleichwertige, aber formal verschiedene Konstruktionen verwendet werden können.

(11) GP innerhalb der pronominalen Possession

\begin{tabular}{|c|c|c|c|}
\hline Possessor & Georgisch & Italienisch & Albanisch \\
\hline $1 \mathrm{Sg}$ & $\mathrm{X}$ & $\mathrm{X}$ & $\mathrm{X}$ \\
\hline $2 \mathrm{Sg}$ & $\mathrm{X}$ & $\mathrm{X}$ & $\mathrm{X}$ \\
\hline $1 \mathrm{Pl}$ & $\mathrm{X}$ & $\mathrm{X}$ & $\mathrm{Y}$ \\
\hline $2 \mathrm{Pl}$ & $\mathrm{X}$ & $\mathrm{X}$ & $\mathrm{Y}$ \\
\hline $3 \mathrm{Sg}$ & $\mathrm{X}$ & $\mathrm{X}$ & $\mathrm{Y} / \mathrm{Z}$ \\
\hline $3 \mathrm{Pl}$ & $\mathrm{Y}$ & $\mathrm{Y}$ & $\mathrm{Y} / \mathrm{Z}$ \\
\hline
\end{tabular}

Während im Georgischen und Italienischen ein Possessor in der 3. Person Plural sich morphosyntaktisch so verhält wie in Kombination mit einem beliebigen appellativischen Possessum außerhalb des Bereichs der Verwandtschaftsverhältnisse (z.B. Italienisch la loro madre mit obligatorischem definiten Artikel und Georgisch mati deda ,ihre Mutter" mit obligatorisch vorangestelltem Possessivpronomen), sind im Albanischen alle pluralischen Possessoren sowie die 3. Person Singular betroffen, wie in (12) mit dem 
nachgestellten Possessivpronomen der 1. Person Plural. D.h. dass Eigenschaften des Possessors Einfluss darauf haben, welche possessivische Konstruktion gewählt wird.

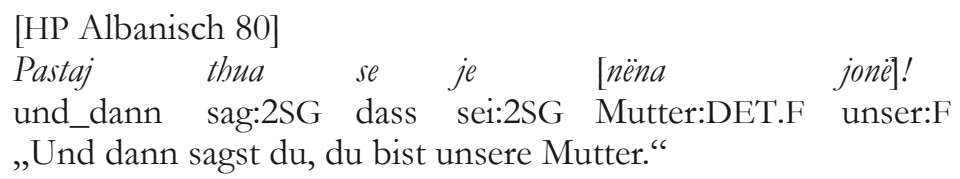

Die Beispiele unter (13), die Übersetzungsäquivalente voneinander sind, reflektieren eine weitere Restriktion, durch die die Wahl der possessivischen Konstruktion bestimmt wird.

(13) Pluralische Possessa

(13a) [HP Deutsch 118]

In welchem Haus sind [deine Brüder]?

(13b) [HP Albanisch 91]

Nëçshtëpi janë [vëllerërit e tu]?

(13c) [HP Italienisch 104]

In quale casa sono [i tuoi fratelli]?

Pluralität ist auch beim Possessum ein Ausschlusskriterium; denn die Possessa verlieren im Georgischen, Albanischen und Italienischen ihr Anrecht auf eine besondere morphosyntaktische Behandlung. Auf pluralische Verwandtschaftstermini als Possessa werden die Regeln angewendet, die für die Masse der Appellativa gelten: im Italienischen $i$ twoi fratelli „deine Brüder" mit definitem Artikel, im Albanischen vëllezęrit e tu mit nachgestelltem Possessivum und im Georgischen (Tamar Reseck persönliche Mitteilung) čemi deida-eb-i „meine Tanten“ mit pränominalem Possessivpronomen. ${ }^{7}$ Man kann von einer Konspiration von Eigenschaften beider Partizipanten sprechen, durch die der Bereich, in dem inalienable Possession sichtbar auftritt, erheblich eingeschränkt wird. Nichtsdestoweniger sind die Distinktionen nicht optional.

Im Gegensatz zum Maltesischen bedient sich keine der drei diskutierten Sprachen eines spezialisierten Morphems, um Inalienabilität zu kodieren. Die Suffigierung des georgischen Possessivums kommt dem maltesischen Muster noch am nächsten. Für Georgisch gilt jedoch wie für das Albanische, dass der Hauptunterschied zwischen inalienabler und alienabler Konstruktion die Linearisierung der Konstituenten betrifft. Im Italienischen ist das alleinige Unterscheidungskriterium die An- bzw. Abwesenheit des definiten Artikels. Beide Verfahren - Änderung der Linearisierung und Setzung/Nichtsetzung des Artikels - sind auch in den inselskandinavischen Sprachen für die Identifikation von GP-Fällen mit maßgebend (Stolz et al. 2008, S. 111-141).

Umgangssprachlich soll auch deida-čem-eb-i $\{$ Tante $\}-\{$ mein $\}-\{\mathrm{PL}\}-\{\mathrm{NOM}\}$ „meine Tanten“ möglich sein (Tamar Reseck persönliche Mitteilung). 
Die Satzpaare unter (14)-(15) übersetzen dieselbe Textpassage wie schon (7)-(10) oben. Sowohl Isländisch als auch Färöisch benutzen zwei verschiedene Konstruktionen in Verbindung mit den Übersetzungsäquivalenten von Deutsch Name und Vater.

(14) Isländisch [HP Isländisch 95]

(14a) Possessum [-Verwandtschaft]

Finnst pér [nafn-ið mitt] snidugt?

find:REF dir Name-DET.N mein:N witzig:N

„Findest du meinen Namen witzig?““

(14b) Possessum [+Verwandtschaft]

[Fadir minn] sagdi mér að ...

Vater mein sag:PRÄT:3SG mir dass

„Mein Vater sagte mir, dass ..."

(15) Färöisch [HP Färöisch 93]

(15a) Possessum [-Verwandtschaft]

So tú heldur [mitt navn] vera stuttligt?

So du mein:2SG mein:N Name sei:INF lustig:N

„Du meinst also, mein Name sei lustig?“

(15b) Possessum [+Verwandtschaft]

[Pápi min $]$ segði, at ...

Vater mein sag:РRÄт:3Sg dass

„Mein Vater sagte, dass ...“

In beiden inselskandinavischen Sprachen ist die Kombination von artikellosem Nomen und nachgestelltem Possessivpronomen das Vorrecht von Verwandtschaftsbeziehungen (Fadir minn = Pápi mín „mein Vater"). Im Unterschied zum Isländischen, das overt definite Appellativa mit dem Possessivpronomen verbindet (nafnið mitt „,mein Name“), ${ }^{8}$ steht diese Möglichkeit dem Färöischen nicht zur Verfügung, das in (15a) stattdessen die pränominale Stellung des Possessivums wählt (mitt navn ,mein Name"). Diese Linearisierung ist relativ häufig bei einer heterogen wirkenden Gruppe von Possessa zu finden, in denen abstrakte Konzepte ein besonderes Gewicht haben (Stolz/Gorsemann 2001, S. 576). Für alienable Possessa vom Typ [+konkret] wird hingegen auf eine dritte Konstruktion zurückgegriffen, die sowohl bei pronominaler als auch bei adnominaler Possession Verwendung findet - die durch hjá „,bei“ eingeleitete Präpositionalphrase, siehe (16).

Thráinsson (2010, S. 93-94) hält Fälle von adnominaler Possession mit overt definitem Possessum im Isländischen für „questionable“ und bestenfalls „dialectally“ für akzeptabel. Ich verzichte an dieser Stelle auf eine vertiefte Diskussion der Gegebenheiten. 
(16) Färöisch

(16a) adnominal [HP Färöisch 86]

Eg havi fingid [tor gomlu skikekjunar bjá Bill, ich hab:1SG bekommen die alt:PL Umhang:DET.PL bei Bill [gamla gandastavin bjá Charlie] og alt:AKK Zauberstab:DET bei Charlie und [ta gomlu rottuna bjá Percy]. die alt:AKK Ratte:DET:AKK bei Percy „Ich habe den alten Umhang von Bill, den alten Zauberstab von Charlie und die alte Ratte von Percy bekommen."

(16b) pronominal [HP Färöisch 90]

Tit hava ikki sad [froskin bjá mar??

ihr hab:PL NEG gesehen Frosch:DET bei mir „Habt ihr nicht meinen Frosch gesehen?“

Während die isländische Version von (16a) mit dem Eigennamen als Possessor noch eine weitere Konstruktion einführt, die erst unter Punkt 3.2 besprochen wird, ist für (16b) das in (14a) illustrierte Muster im Isländischen anzuwenden: halakörtuna mina „meinen Frosch“.

Die herausgehobene Stellung der Verwandtschaftstermini setzt sich im possessivischen System der beiden inselskandinavischen Sprachen auch im Bereich der prädikativen Possession fort. Isländisch macht von drei verschiedenen Possessionsverben Gebrauch, nämlich eiga, hafa und vera með, von denen die ersten beiden auch im Färöischen (als eiga und hava) verwendet werden (Stolz et al. 2008, S. 153-214). Für diesen Abschnitt ist wichtig, dass ausschließlich eiga eingesetzt wird, wenn die Possessum-NP einen Verwandtschaftsterminus beinhaltet. In den deutschen Beispielsätzen unter (17) macht es keinen Unterschied, welcher semantischen Klasse das Possessum angehört; das Possessionsverb ist immer haben. Die äquivalenten Satzpaare aus dem Isländischen (18) und Färöischen (19) zeigen jedoch einen Gegensatz zwischen hafa/hava, das für Possessa außerhalb des Verwandtschaftsbereichs gilt, und eiga, das mit Verwandtschaftstermini zu verwenden ist.

(17) Deutsch

(17a) Possessum [-Verwandtschaft]

Sie [haben die Augen] Ihrer Mutter.

(17b) Possessum [+Verwandtschaft]

Die Dursleys [hatten einen kleinen Sohn] namens Dudley ...

(18) Isländisch

(18a) Possessum [-Verwandtschaft]

Dú [hefur augun [hennar [módur Dinnar]]].

du hab:2SG Auge:DET:PL sie:GEN Mutter dein:GEN.F

„Du hast die Augen deiner Mutter.“ 
(18b) Possessum [+Verwandtschaft]

Dursleybjónin [áttu lítinn dreng

Dursley_Ehepaar:DET hab:PRÄT:3PL klein:AKK Junge

ad nafni Dudley...

zu Name:DAT Dudley

„Das Eheparr Dursley hatte einen kleinen Jungen namens Dudley ..."

(19) Färöisch

(19a) Possessum [-Verwandtschaft]

Tú [hevur [eyguni i [mammu tini]]].

du hab:2SG Auge:DET.PL in Mutter:DAT dein:DAT „Du hast die Augen deiner Mutter.“

(19b) Possessum [+Verwandtschaft] [HP Färöisch 5]

Tey [áttu ein son], sum at Dudley... sie hab:PRÄT:PL ein(AKK) Sohn(AKK) der heiß:PRÄT Dudley „Sie hatten einen Sohn, der Dudley hieß ..."

Die Domäne von eiga ist in den beiden inselskandinavischen Sprachen nicht ganz deckungsgleich. Jenseits der Verwandtschaftsverhältnisse wird dieses Possessionsverb in Kontexten verwendet, die im Deutschen Übersetzungen mit besitzen nahelegen, also De-Jure-Possession betreffen, worauf ich nicht weiter eingehen kann.

Den inselskandinavischen Befund fasse ich in der Synopse unter (20) zusammen. Verwandtschaftstermini sind in der pronominalen und prädikativen Possession mit grammatisch relevanten morphosyntaktischen Konsequenzen verbunden, da sie Konstruktionen verlangen, die mit anderen Possessa normalerweise nicht auftreten.

(20) Konstruktionen mit possedierten Verwandtschaftstermini im Inselskandinavischen

\begin{tabular}{|c|c|c|c|c|c|c|}
\hline Typ & Syntax & \multicolumn{2}{|c|}{ Isländisch } & \multicolumn{2}{|c|}{ Färöisch } & $\begin{array}{c}\text { Possessum } \\
\text { Verwandtschaft }\end{array}$ \\
\hline \multirow{4}{*}{ attributiv } & \multirow{2}{*}{ pronominal } & \multicolumn{2}{|c|}{$\mathrm{N}+$ Pro } & \multicolumn{2}{|l|}{$\mathrm{N}+$ Pro } & + \\
\hline & & \multicolumn{2}{|c|}{$\mathrm{N}_{[+ \text {definit }]}+$ Pro } & Pro $+\mathrm{N}$ & N bjá Pro & $\neg$ \\
\hline & \multirow{2}{*}{ adnominal } & \multirow{2}{*}{\multicolumn{2}{|c|}{$\mathrm{N}+\mathrm{N}$}} & \multirow{2}{*}{\multicolumn{2}{|c|}{$\mathrm{N}$ bjá $\mathrm{N}$}} & + \\
\hline & & & & & & $\neg$ \\
\hline \multirow{2}{*}{\multicolumn{2}{|c|}{ prädikativ }} & \multicolumn{2}{|l|}{ eiga } & \multicolumn{2}{|l|}{ eiga } & + \\
\hline & & bafa & vera meठ & \multicolumn{2}{|l|}{ hava } & $\neg$ \\
\hline
\end{tabular}


Mit allen zuvor in diesem Abschnitt vorgestellten Sprachen teilen Isländisch und Färöisch die Eigenschaft, dass possedierte Verwandtschaftstermini in der pronominalen Possession grammatisch relevante Auswirkungen haben. Anders als Georgisch, Albanisch und Italienisch weisen die inselskandinavischen Sprachen aber keine weiteren Spaltungen innerhalb des Paradigmas auf und kennen auch keine Restriktionen hinsichtlich des Numerus des Possessums. Sowohl Isländisch als auch Färöisch zeigen, dass die prädikative Possession ebenfalls eine distinkte Konstruktion erfordert, wenn das Possessum ein Verwandtschaftsterminus ist. Dies setzt sie genauso von den übrigen vier Sprachen ab wie das Maltesische mit dem status constructus, das als einzige in diesem Abschnitt besondere morphosyntaktische Strukturen für possedierte Verwandtschaftstermini in adnominaler Possession vorsieht. Unter (21) werden die sechs Sprachen zusammenfassend einander gegenübergestellt. Das X bezeichnet einen Bereich, in dem sich possedierte Verwandtschaftstermini grammatisch relevant verhalten. Die hellgraue Schattierung identifiziert die Bereiche, in denen noch weitere Restriktionen wirksam sind. Dunkelgrau sind diejenigen Zellen unterlegt, in denen Verwandtschaftsbezeichnungen kein grammatisches Privileg besitzen.

(21) Synopse: Grammatische Relevanz possedierter Verwandtschaftstermini

\begin{tabular}{|c|c|c|c|c|c|c|}
\hline Typ & Georgisch & Albanisch & Italienisch & Maltesisch & Isländisch & Färöisch \\
\hline Pronominal & $\mathrm{X}$ & $\mathrm{X}$ & $\mathrm{X}$ & $\mathrm{X}$ & $\mathrm{X}$ & $\mathrm{X}$ \\
\hline Adnominal & & & & $\mathrm{X}$ & & \\
\hline prädikativ & & & & & $\mathrm{X}$ & $\mathrm{X}$ \\
\hline
\end{tabular}

\subsubsection{Körperteile}

Die zweite Kategorie inalienabler Possessa bilden Körperteilbezeichnungen. Im Maltesischen verhalten sich diese analog zu den Verwandtschaftstermini, d.h. dass sie die Possessorsuffixe bei pronominaler Possession verlangen (21a) und bei adnominaler Possession im status constructus (21b) stehen.

(21) Maltesisch

(21a) pronominale Possession [Ǵahan 17]

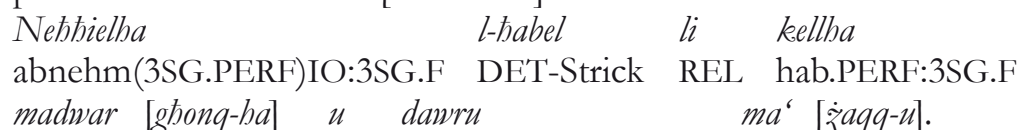

um Hals-3SG.F und dreh(3SG.Perf)3SG.M mit Bauch-3SG.M „Er nahm ihr den Strick ab, den sie um den Hals trug, und er band ihn sich um den Bauch." 
(21b) adnominale Possession [Ġahan 52]

$\begin{array}{lllll}\text { Bid-daqqa, } & \text { il-gebla } & l i & \text { kienet } & \text { flok } \\ \text { mit:DET-Schlag } & \text { DET-Stein } & \text { REL } & \text { sei:PERF.3SG.F } & \text { an_Stelle } \\ \text { [ras } & \text { Gahan] } & \text { saret } & & \text { trab. } \\ \text { Kopf } & \text { Gahan } & \text { werd:PERF.3SG.F } & \text { Staub } & \end{array}$
„Durch den Schlag wurde der Stein, der an Stelle von Gaћans Kopf (dort) war, zu Staub.“

Während im Albanischen, Italienischen und Georgischen Körperteilbezeichnungen keine grammatischen Privilegien in der Possession aufweisen, spielen sie im nordgermanischen Bereich genauso wie im Maltesischen eine herausragende Rolle bei der Gestaltung der possessivischen Morphosyntax. Dies ist besonders ausgeprägt im Färöischen und Isländischen, während in den festlandskandinavischen Sprachen ein höheres Maß an Variation zu beobachten ist.

Wo im Maltesischen für beide Klassen von inalienablen Possessa die gleichen Konstruktionsmuster verwendet werden, gelten im Isländischen und Färöischen für possedierte Körperteilbezeichnungen andere Konstruktionen als für possedierte Verwandtschaftstermini. In beiden Sprachen wird der Possessor als präpositionales Attribut an das Possessum angeschlossen, wobei die räumlichen Präpositionen $a$, an, auf“ und $\hat{\imath}$, in “, die in nicht ganz überlappungsfreier Verteilung auf die Ausdrücke für possedierte äußere und innere Körperteile folgen. Diese Regel umfasst sowohl die adnominale als auch die pronominale Possession. Die Übersetzungsäquivalente der deutschen Genitivkonstruktion (22a) und der Konstruktion mit dem Possessivpronomen (22b) sind im Isländischen (23) und Färöischen (24) jeweils syntaktisch gleich aufgebaut.

(22) Deutsch

(22a) adnominal [HP Deutsch 142]

Harrys Beine waren wieder bleischwer ...

(22b) pronominal [HP Deutsch 79]

... sein linkes Auge zuckte

(23) Isländisch

(23a) adnominal [HP Isländisch 112]

[Fcturnir á Harry] voru aftur orơnir

Fuß:DET:PL auf Harry sei.PRÄT:3PL wieder geworden

blypungir...

bleischwer:PL

„Harrys Füße waren wieder bleischwer geworden ...“

(23b) pronominal [HP Isländisch 62]

... og annad [augad i bonum] kipptist til

... und anderes Auge:DET in ihm zuck:PRÄT:REF zu 


$$
\begin{aligned}
& \text { i sifellu } \\
& \text { in_einem_fort } \\
& \text { „... und sein anderes Auge zuckte in einem fort.“ }
\end{aligned}
$$

(24) Färöisch

(24a) adnominal [HP Färöisch 110]

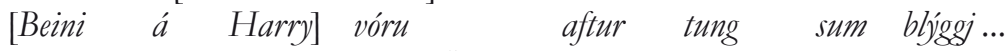
Bein:PL auf Harry sei:PRÄT:PL wieder schwer wie Blei „Harrys Beine waren wieder schwer wie Blei ...“

(24b) pronominal [HP Färöisch 62]

[Annad eygad $i$ bonum] skrykti um

Anderes Auge:DET in ihm zuck:PRÄT:3SG über eygnalokini.

Augenlid:DET:DAT

„Sein anderes Auge zuckte am Augenlid.“

Im Gegensatz zum Färöischen, das sich bei Körperteilpossession stets auf hava als Possessionsverb stützt, teilen Isländisch und Maltesisch noch eine weitere Eigenschaft. In beiden Sprachen wirken sich possedierte Körperteile auch in der prädikativen Possession strukturell aus. Dies trifft besonders auf das Isländische zu, da hier recht konsequent das Possessionsverb vera med $X$ wörtlich „mit X sein“ Verwendung findet. Das Beispiel unter (25) illustriert dessen Gebrauch im Satzzusammenhang.

(25) Isländisch [HP Isländisch 92]

Hún var med mikid brunt hár og stórar sie sein:PRÄT mit viel:N braun:N Haar und groß:PL framtennur. Vorderzahn:PL

„Sie hatte einen üppigen braunen Haarschopf und recht lange Vorderzähne."

Das in (17a) mit possedierten Körperteilen verwendete isländische Possessionsverb hafa ist in meinen Korpustexten nur ein minder frequenter Konkurrent von vera með $X$, das in gut $90 \%$ aller Fälle von prädikativ-possedierten Körperteilen eingesetzt wird. Daher heißt es auch alternativ zu (18a) in Hagrids Worten in (26):

(26) Isländisch [HP Isländisch 44]

... en [pú ert med [angun [hennar mömmu ... aber du sei:2SG mit Auge:DET:PL sie:GEN Mutter:GEN pinnar]]].

deiner

„,.. aber du hast die Augen deiner Mutter.“ 
Den nur oberflächlich parallelen maltesischen Fall bespreche ich in den folgenden Abschnitten. Die Tabelle unter (27) fasst für den Bereich der attributiven Possession die Regelungen der inalienablen Possession im Maltesischen und den beiden inselskandinavischen Sprachen schematisch zusammen. Die Buchstabenkürzel stehen für verschiedene Konstruktionen.

(27) Synopse: NP-interner Ausdruck von Inalienabilität

\begin{tabular}{|c|c|c|c|c|}
\hline \multirow[t]{2}{*}{ Sprache } & \multirow{2}{*}{$\begin{array}{l}\text { Attributive } \\
\text { Possession }\end{array}$} & \multicolumn{2}{|c|}{ inalienabel } & \multirow{2}{*}{ alienabel } \\
\hline & & Verwandtschaft & Körperteil & \\
\hline \multirow{2}{*}{ Maltesisch } & adnominal & \multicolumn{2}{|c|}{$\mathrm{X}$} & \multirow[t]{2}{*}{ Z } \\
\hline & pronominal & \multicolumn{2}{|c|}{$\mathrm{Y}$} & \\
\hline \multirow{2}{*}{ Inselskandinavisch } & adnominal & A & \multirow{2}{*}{$\mathrm{C}$} & $\mathrm{D}$ \\
\hline & pronominal & B & & $\mathrm{E}$ \\
\hline
\end{tabular}

\subsubsection{Physisch-mentale Zustände}

Mit diesem Abschnitt begebe ich mich außerhalb des üblicherweise in der crosslinguistisch ausgerichteten Possessionsforschung gesteckten Rahmens. An der Typologie der Ausdrücke für physisch-mentale Zustände wird im Rahmen der Erforschung von experientiellen Konstruktionen gearbeitet, wobei possessivischen Konstruktionen insgesamt nur eine Nebenrolle zuzukommen scheint (Verhoeven 2007, S. 83-86). Im Deutschen können Gefühle (Ich habe Angst./Meine Angst ist groß.), Krankheiten (Ich babe Grippe./ Meine Grippe ist ansteckend.), körperliche Erfahrungen (Ich babe Hunger./Mein Hunger ist groß.) usw. genauso possediert werden wie beliebige andere Konzepte. Ähnlich unspektakulär fällt das Ergebnis für viele andere Sprachen Europas aus. In vielen europäischen Sprachen sind physisch-mentale Zustände mögliche Possessa; jedoch werden sie nicht in allen Fällen analog zum Deutschen wie beliebige sonstige Possessa behandelt.

Das Maltesische sieht vor, dass in der attributiven Possession auf den possessivischen Relativsatz (Stolz im Druck) zurückgegriffen wird, wenn das Possessum zur Klasse der physisch-mentalen Zustände gehört. Dies gilt adnominal (28a) genauso wie pronominal (28b).

(28) Maltesisch

(28a) adnominal [Wenzu 147]

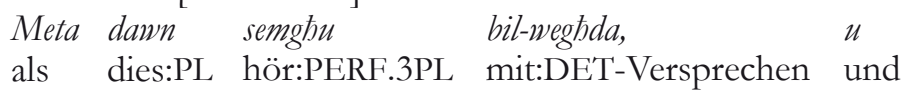

[bil-biża' li kellu Wenzu] ...

mit:DET-Angst REL hab.Perf:3SG.M Wenzu

„Als diese von dem Versprechen und von Wenzus Angst hörten, ...“ 
(28b) pronominal [Gahan 117]

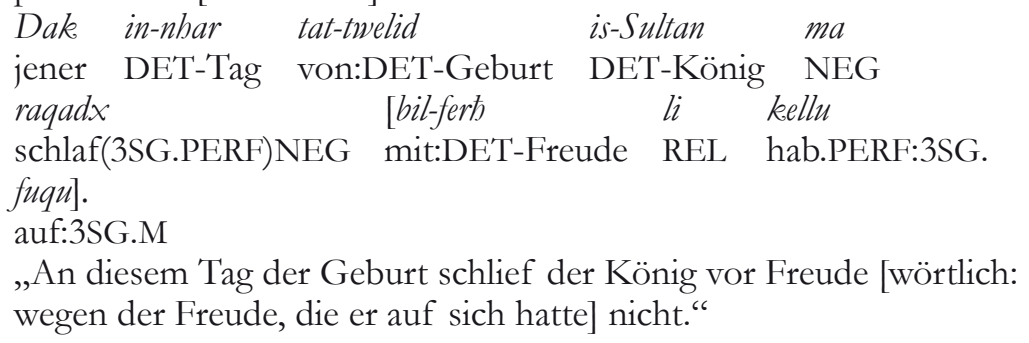

Es wird auf diese Weise vermieden, die anderen in der NP üblichen Konstruktionsweisen, z.B. mit der durch $t a^{\prime}$,,von“ eingeleiteten PP, zur Possessormarkierung einzusetzen. Da die Relativsatzstrategie ein Possessivverb im Relativsatz selber erfordert, kann der Eindruck entstehen, die NPn, die physisch-mentale Zustände ausdrücken, seien problemlos mit diesen Verben in der prädikativen Possession kombinierbar. Diese Hypothese trifft auch auf den ersten Blick zu; bei genauerem Hinsehen ist jedoch deutlich zu erkennen, dass es für prädikativ-possedierte physisch-mentale Zustände eine mit manchen Possessa vorzugsweise, mit anderen Possessa nur gelegentlich genutzte alternative Konstruktion gibt (29).

(29) Maltesisch

(29a) [-physisch-mental] [Gahan 25]

Jien [ghandi l-berritta] biex nghattiha

ich hab:1SG DET-Mütze womit 1SG.IMPERF:bedeck:3SG.F

l-qargha ...

DET-Glatze

„Ich habe die Mütze, womit ich die Glatze bedecke ..."

(29b) [+physisch-mental] [Malta 498]

Kienet ghadha bin-nghas

sei:PERF.3SG.F noch:3SG.F mit:DET-Schlaf

„Sie war noch schläfrig."

In (29a) ist das Possessum ein Kleidungsstück (il-berritta „die Mütze“) und nimmt daher das Possessionsverb ghand-/kell-/ikoll- „haben“. Dahingegen ist in (29b) die Rede von einer körperlichen Erfahrung (in-ngtas „,der Schlaf/ die Schläfrigkeit"). Dieses Possessum verbindet sich besser mit dem prädikativen Possessionsausdruck KOPULA + bi ,sein mit“.

Vergleichbare Konstruktionen, in denen eine Kopula und eine Präposition, die Deutsch mit übersetzt, auftreten, finden sich in der prädikativen Possession auch in anderen Sprachen Europas (Stolz 2001, S. 344), besonders aber im Isländischen und Portugiesischen. Die schon von den possedierten Körperteilen her bekannte isländische Konstruktion vera meઇ $X$ wird beispielsweise verwendet, wenn man ausdrücken will, dass jemand eine Krankheit „hat“" (30). 
(30) Isländisch [HP Isländisch 256]

Hann var einn med bradilegan bausverk. er sei:PRÄT.3SG noch mit furchtbar:AKK Kopfweh „Er hatte immer noch grässliches Kopfweh.“

Im Portugiesischen kommt von den beiden Kopulae nur estar „sein“ in Betracht, das vorübergehende Zustände und räumliche Verortung ausdrückt. Das Possessum wird durch die Präposition com „mit“ eingeleitet (Endruschat 2007, S. 211). Eine typische Gebrauchsweise illustriert (31b); estar com medo stimmt mit Deutsch Angst haben inhaltlich überein. In (31a) wird der Einsatz des ansonsten dominanten Possessionsverbs ter „haben“ gezeigt.

(31) Portugiesisch

(31a) [-physisch-mental] [HP Portugiesisch 9]

$\begin{array}{llllll}\text { Os } & \text { Dursleys } & \text { [tinham } & \text { um } & \text { filho } & \text { pequeno] } \\ \text { DET:PL } & \text { Dursleys } & \text { hab:PRÄT:3PL } & \text { INDEF } & \text { Sohn } & \text { klein } \\ \text { chamado } & \text { Dudley ... } & & & & \\ \text { genannt } & \text { Dudley } & & & \\ \text { "Die Dursleys hatten einen kleinen Sohn, genannt Dudley ...“ }\end{array}$

(31b) [+ physisch-mental] [HP Portugiesisch 54]

Nunca fizeste acontecer
nie mach:PERF:2SG sich_ereign:INF Sache:PL
estranhas quando [estavas $\quad$ com medo] ou zangado?
seltsam:PL als sei:IMPERF:2SG mit Angst oder erzürnt
„Hast du niemals seltsame Dinge geschehen lassen, wenn du Angst
hattest oder zornig warst?“

Drei Sprachen zeigen GP im Zusammenhang mit Possessa, die physischmentale Zustände bezeichnen. Dabei ist nur im Maltesischen auch die attributive Possession strukturell betroffen, in allen drei Sprachen ist aber die prädikative Possession sensitiv für Unterschiede zwischen physisch-mentalen Zuständen und beispielsweise konkreten Possessa.

\subsection{Vom Possessum (teilweise) unabhängige GP}

Physisch-mentale Zustände sind gewöhnlich temporärer Natur und nicht chronisch. Ihre zeitliche Begrenztheit trägt dazu bei, sie gleich in zweifacher Hinsicht vom possessivischen Prototyp zu distanzieren. Zum einen sind physisch-mentale Zustände normalerweise außerhalb der Kontrolle des Experiencers/Possessors und zum anderen verhindert ihre Vergänglichkeit, dass die possessivische Relation Permanenz zeigt. Es ist daher nicht überraschend, wenn die im vorangehenden Abschnitt 3.1.3 einge- 
führten Konstruktionen nicht nur mit physisch-mentalen Possessa in Gebrauch sind, sondern sich auch für den Ausdruck von temporären bzw. vom Possessor nicht im eigentlichen Sinne kontrollierten possessiven Relationen anbieten.

Damit komme ich zur Besprechung von GP, die nicht primär von der semantischen Klasse des Possessums abhängig sind. Maltesisch verwendet die Konstruktion KOPULA + bi speziell dann, wenn zeitlich begrenzte Beziehungen zwischen Possessor und Possessum gegeben sind, wie etwa beim Tragen von bestimmten Kleidungsstücken zu einem bestimmten öffentlichen Anlass (32).

(32) Maltesisch [Malta 463]

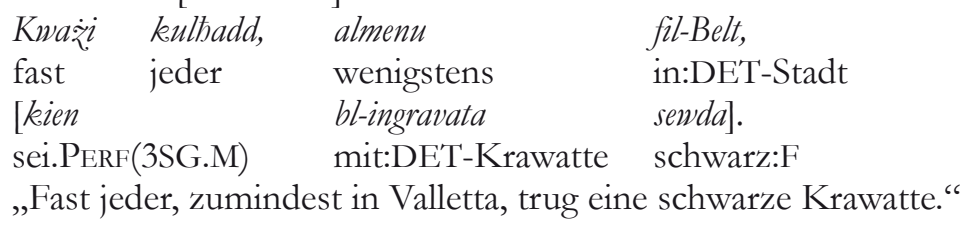

Hier wird nicht darauf abgehoben, dass ein juristisches Besitzverhältnis zwischen Possessor und Possessum besteht, sondern dass beide Partizipanten zu einem gegebenen Zeitpunkt miteinander zu tun hatten.

Der isländische Satz in (33) beschreibt wiederum einen zeitlich beschränkten Ausschnitt, nämlich die aktuelle Possession. Die Frage ist, ob Harry zum Äußerungszeitpunkt den zuvor eingesteckten Brief bei sich trägt, ob er ihn gerade in diesem Augenblick dabei hat.

(33) Isländisch [HP Isländisch 59]

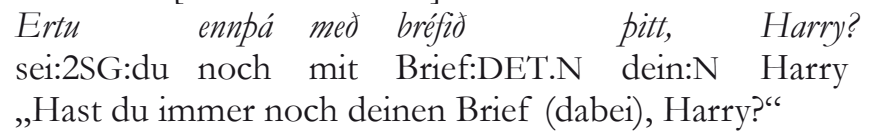

Ganz ähnlich gestaltet sich das portugiesische Beispiel unter (34). Die sich ständig verändernden Punktestände am abschließenden Wettkampftag stellen den Hintergrund für die Feststellung, dass das Haus Gryffindor zu einem gegebenen Zeitpunkt über einhundert Punkte mehr als noch kurze Zeit zuvor verfügte. Auch dies ist nur eine ephemere Zwischenstandsmeldung, da sich die Punktezahl umgehend weiter erhöht.

Portugiesisch [HP Portugiesisch 252]
Estavam com cem pontos a mais.
sei:IMPERF:3PL mit hundert Punkt:PL an mehr
„Sie hatten (jetzt gerade) hundert Punkte mehr.“

So erklärt sich auch die unterschiedliche Wahl des prädikativen Possessionsausdrucks in (35) und (31b). 
Portugiesisch [HP Portugiesisch 52]

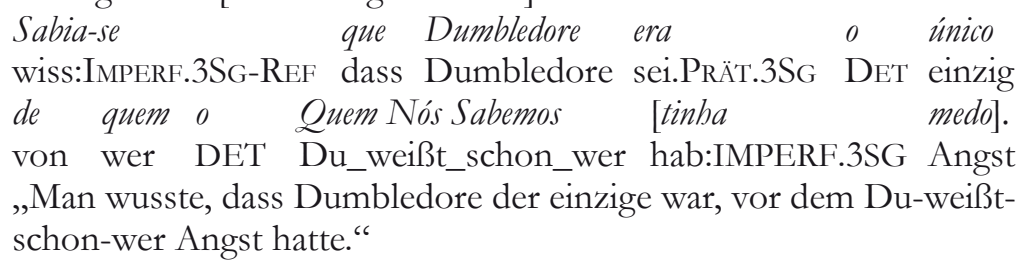

In (31b) werden momentane, vorübergehende Angstzustände angesprochen, sodass die Verwendung der Konstruktion KOPULA + com angemessen ist. Die Angst in (35) hingegen ist situationsunabhängig und von Dauer. Daher wird das für den Ausdruck permanenter Relationen geeignete Possessionsverb ter eingesetzt.

In diesen und ähnlichen Fällen bedient man sich im Deutschen bei Situationen aktueller oder physischer Possession z.T. abgeleiteter Formen des Possessionsverbs haben, also anhaben, umbaben, dabeibaben usw., die allerdings oft durch einfaches haben ersetzt werden können. GP ist im Deutschen daher nur eine unterdrückbare Möglichkeit, während sich im Maltesischen, Isländischen und Portugiesischen der temporäre Charakter der possessivischen Relation stärker auf die Wahl der Konstruktion auswirkt, ohne allerdings diese zu $100 \%$ zu determinieren.

Die unter (2) vorgestellte Form von GP im Deutschen, durch die den Eigennamen eine Sonderstellung unter den Possessorausdrücken zugewiesen werden kann, hat recht genaue Parallelen im Niederdeutschen und Niederländischen. Strukturell anders gelagert ist der im Färöischen und Isländischen belegte Gebrauch von Possessivpronomina in Kombination mit Eigennamen. Das isländische Beispiel (36) enthält drei Belege für diese Konstruktion.

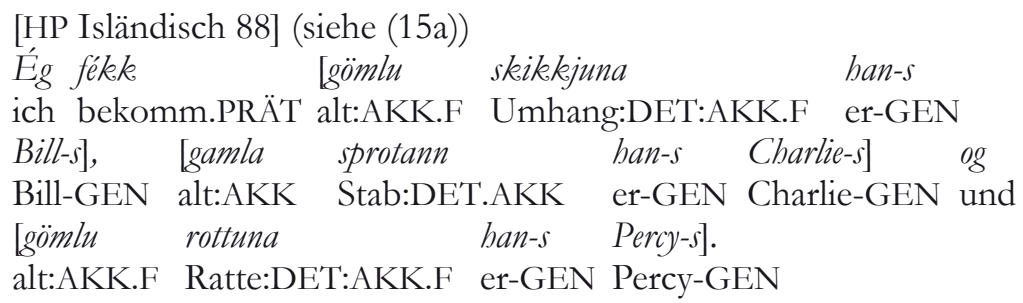

„Ich habe den alten Umhang von Bill, den alten Zauberstab von Charlie und die alte Ratte von Percy bekommen."

Die Possessoren sind durch ihre Eigennamen Bill, Charlie und Percy identifiziert, die jeweils noch das genitivische Flexiv $-s$ tragen. Trotz dieser eindeutigen morphologischen Kennzeichnung können die Eigennamen jedoch nicht direkt mit der Possessum-NP kombiniert werden. Ihnen muss das ebenfalls genitivisch flektierte Possessivpronomen - in diesem Fall jeweils 
hans „sein(er)" - vorangehen. Als Eigennamen werden auch Verwandtschaftstermini wie mamma „Mutter“ (wie in (18a) und (27)) behandelt. Die morphosyntaktische Komplexität der possessivischen Konstruktion wird durch das obligatorische Possessivpronomen erhöht.'

Hohe Belebtheit bei den Possessoren ${ }^{10}$ und Temporalität bei den possessivischen Relationen tragen also zur weiteren Ausdifferenzierung des Systems der GP in Europa bei. Eine feste Bindung an die Semantik des Possessums allein ist daher bei GP nicht gegeben, wobei bestimmte Possessa - eben physisch-mentale Zustände - mit temporärer Possession gut zusammenpassen, da sie selber nur eine zeitlich begrenzte „Lebensdauer“ haben.

\subsection{Störfaktoren}

Dass man überhaupt von einem System sprechen kann, in das GP maßgeblich eingebunden ist, erschließt sich für einige der hier untersuchten und andere Sprachen erst bei genauerem Hinsehen. Der eher flüchtige Blick auf die Daten kann in die Irre führen, weil es mächtige Bedingungen gibt, unter denen die oben angesprochenen Distinktionen neutralisiert werden. Diese Bedingungen treten im Textzusammenhang relativ häufig auf, sodass selbst bei einer korpusgestützten Datensammlung oberflächlich der Eindruck freier Austauschbarkeit von synonymen Konstruktionen entstehen kann. Die Faktoren, die hierzu hauptsächlich beitragen, sind semantische Differenzierung, Pragmatik und morphosyntaktisches Gewicht, deren Einflussnahme auf die Wahl der possessivischen Konstruktion ich nachstehend auszugsweise illustriere.

Während im Albanischen, Georgischen und Italienischen GP in der attributiven Possession ausschließlich bei possedierten Verwandtschaftsbezeichnungen auftritt, umfasst die Domäne der GP im Maltesischen und den beiden inselskandinavischen Sprachen zusätzlich nicht nur Körperteilbezeichnungen, sondern auch eine ansehnliche Anzahl von so genannten individuellen Konzepten, die in keine der sonstigen Kategorien der Inalienabilität fallen. Für das literarische Maltesische sind entsprechende Konzepte dutzendfach belegt (Stolz et al. 2008, S. 52-54). Dazu gehören z.B. Bezeichnungen für die Phasen im Leben eines Menschen wie tfuliti „,meine Kindheit" (von tfulija „Kindheit“). Für Isländisch und Färöisch sind die Belege ebenfalls keine statistische Nebensächlichkeit. Im Isländischen verhalten

Für weitere auf den Possessor bezogene GP (z.B. den färöischen possessivischen Akkusativ und den sekundären Genitiv) siehe Stolz/Gorsemann (2001).

10 Lutz Gunkel (persönliche Mitteilung) weist für das Deutsche in diesem Zusammenhang darauf hin, dass Personalpronomina deiktisch nicht zur Referenz auf unbelebte Gegenstände verwendet werden können. Diese Restriktion überträgt sich auch auf die Possessiva. Beispiel: [Verweis auf einen Mann] Ich meine SEINE Krawatte. vs. [Verweis auf eine Tür] ??Ich meine IHRE Farbe. 
sich in der attributiven Possession Possessa, die das Merkmal [-konkret] tragen, überwiegend wie Verwandtschaftstermini, d.h. dass beispielsweise wieder Ausdrücke, die sich auf Lebensabschnitte beziehen, als Possessum keinen definiten Artikel tragen dürfen: i lifi peirra ,in ihrem Leben“ und nicht *i lifinu peirra (ebd., S. 124).

Für eine ganze Reihe von Termini, die individuelle Konzepte vertreten, ist nachweisbar, dass sie sich in possessivischen Konstruktionen sowohl wie typische inalienable Possessa als auch wie alienable Possessa verhalten können. Was auf den ersten Blick wie Beliebigkeit aussieht, erweist sich rasch als semantisch determinierte Wahl der passenden Konstruktion. Denn durch die verschiedenen possessivischen Konstruktionen können unterschiedliche Lesarten und Bedeutungen von Substantiven differenziert werden. Dabei gilt die Regel, dass metaphorische Lesarten mit der inalienablen Possession assoziiert werden. Wenn ein Possessum zwei Konstruktionsweisen zulässt, zeigt die inalienable Konstruktion ein engeres (z.T. emotionales) Verhältnis des Possessors zum Possessum an. Ein schlagendes Beispiel ist das maltesische Substantiv dar „Haus“, das in (37) mit seinen beiden possessivischen Gebrauchsweisen vertreten ist.

(37) Maltesisch

(37a) alienabel [+konkret] [Malta 242]

... biex tinkoraggixixi l-propretarji

... damit 3SG.F.IMPERF:ermutig DET-Besitzer:PL

jimmoderniżzaw [id-djar taghbom].

3PL.IMPERF:modernisier:PL DET-Haus.PL von:3PL

„,... damit sie die Besitzer dazu ermutige, ihre Häuser zu modernisieren."

(37b) inalienabel [ \pm konkret] [Malta 45]

... illum uliedhom qeghdin jarmaw

... und heute Kind.PL:3PL PROG:PL 3PL.IMPERF:ausstatt:PL [djar-hom] wisq abjar.

Haus.PL-3PL sehr besser

„,... und heute statten ihre Kinder ihre Häuser viel besser aus.“

In (37a) geht es um den wirtschaftlichen Aspekt des Hausbesitzes. Es spielt keine Rolle, ob die Hausbesitzer auch die zu modernisierenden Gebäude bewohnen. Die Häuser werden als veräußerliche Objekte konzipiert. Daher wird die Konstruktion für alienable Possessa verwendet. In (37b) hingegen geht es um die Häuser, die den Bewohnern eine Heimstatt bieten, den Familiensitz bilden und zu denen die Possessoren daher in einer engen Beziehung stehen. In diesem Fall wird die Konstruktion für inalienable Possessa gewählt. Ähnlich (aber in wesentlich geringerem Umfang) verhalten sich einige der individuellen Konzepte im Isländischen (ebd., S. 125-126). Ein 
Teil der zu beobachtenden Variation bei der Wahl der possessivischen Konstruktionen ist also eindeutig semantisch motiviert und kann als regelgeleitet verstanden werden. Dass auch darüber hinaus die Variation nicht völlig willkürlich verläuft, zeigen die folgenden Ausführungen.

Possessivische Konstruktionen beinhalten Bezüge auf drei Größen: den Possessor, das Possessum und die Relation im engeren Sinne. Will man eine dieser Komponenten in den Vordergrund stellen, also pragmatisch fokussieren, kann es zu morphosyntaktischen Konsequenzen kommen. Im Maltesischen können beispielsweise die Possessoraffixe nicht den Akzent tragen, sodass sie sich nicht für Kontrastfokuskonstruktionen eignen, bei denen der Possessor hervorgehoben wird. Als Träger des Fokusakzents kommt hingegen die flektierte Präposition $t a^{\prime}$ in Frage, die ansonsten in der alienablen Possession Verwendung findet. ${ }^{11}$ Die Tabelle in (38) führt eine kleine Auswahl von typischen Minimalpaaren an, die die vom Skopus des Fokus abhängige Wahl der possessivischen Konstruktion veranschaulichen.

Maltesisch

\begin{tabular}{|c|c|c|c|}
\hline \multicolumn{4}{|c|}{ Fokus auf } \\
\hline \multicolumn{2}{|c|}{ Possessum } & \multicolumn{2}{|c|}{ Possessor } \\
\hline dmiri & „meine Pflicht" & $i d-d m i r$ tieghi & „meine Pflicht" \\
\hline pajjiżna & „unser Land“" & il-pajjiżtaghna & „unser Land“ \\
\hline habibi & „mein Freund“" & il-万abib tieghi & „mein Freund“ \\
\hline żghożiti & „meine Jugendzeit" & 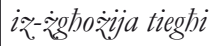 & „meine Jugendzeit“ \\
\hline dari & „mein Haus/Heim“" & id-dar tiegti & „meinHaus/Heim“ \\
\hline \multicolumn{2}{|c|}{ Konstruktion für Inalienabilität } & \multicolumn{2}{|c|}{ Konstruktion für Alienabilität } \\
\hline
\end{tabular}

Analog zum maltesischen Fall wird in den beiden inselskandinavischen Sprachen der Einsatz verschiedener possessivischer Konstruktionen je nach Fokus geregelt. So setzt das Isländische bei Possessorfokus unabhängig von der semantischen Klasse, zu der das Possessum gehört, die Konstruktion ein, in der das Possessivpronomen pränominal steht. Da in dieser Konstruktion grundsätzlich die Possessum-NP ohne Definitheitszeichen steht, werden alle Distinktionen der Alienabilitätskorrelation neutralisiert.

11 Für einige Sprecher des Maltesischen ist diese $t a$-Konstruktion überdies die einzige, die unter Fokus verwendet werden kann - und zwar unabhängig davon, welcher Partizipant hervorgehoben wird (Maris Camilleri persönliche Mitteilung). Die inalienable Konstruktion wäre dann pragmatisch „neutral“. Diese Sichtweise wird nicht von allen muttersprachlichen Linguisten geteilt (Ray Fabri persönliche Mitteilung). 
(39) Isländisch

(39a) Possessorfokus [HP Isländisch 33]

...petta er [mitt bréf $]$.

... dies sei.3SG mein:N Brief

„... das ist mein Brief!“

(39b) neutral/Possessumfokus [HP Isländisch 34]

Hvar er [bréfid mitt?

wo sei.3SG Brief:DET.N mein:N

„Wo ist mein Brief?"

Im Kontext von (39a) wird darum gerungen, wem der umkämpfte Brief eigentlich gehört. Daher wird die Voranstellung des Possessivpronomens gewählt. Die Frage in (39b) fokussiert hingegen den Brief, dessen Verbleib erfragt wird. Daher steht das Possessivpronomen in seiner erwarteten Position rechts vom Possessum-Nomen. Die im Isländischen, Färöischen und Maltesischen mit morphosyntaktischen Mitteln korrelierenden Fokusunterschiede werden im Deutschen nur über suprasegmentale Verfahren markiert. Die possessivischen Konstruktionen bleiben auf morphosyntaktischer Ebene unverändert.

Zum Abschluss dieses Unterkapitels weise ich auf einen dritten Störfaktor hin, der in der Mehrzahl meiner Beispielsprachen dafür sorgt, dass die possessivischen Distinktionen immer wieder verdeckt werden. Es geht um das syntaktische Gewicht der NPn, die an den possessivischen Konstruktionen beteiligt sind. Aus Platzgründen begnüge ich mich mit einem Streiflicht auf die Komplexität der Possessum-NP. Die formalen Unterschiede zwischen inalienabler und alienabler Possession werden zu Gunsten der Konstruktionsweise der letztgenannten aufgehoben, wenn das possedierte Nomen von zusätzlichen Modifikatoren begleitet wird. Adjektivische Attribute sind für diesen Effekt das Paradebeispiel. Dies reflektiert das maltesische Satzpaar in (40).

(40) Maltesisch

(40a) leichte NP [Saltniet 92]

$\mathrm{Hi}$ reghxet fertana, imwerwra

sie erröt:3SG.F.PERF glücklich:F aufgewühlt:F

minn [głajnejn missier-ba] $u$ minn [głajnejn Ganni]...

von Auge:PL Vater-3SG.F und von Auge:PL Ganni

„Sie errötete glücklich, durch die Blicke ihres Vaters und die Blicke

Gannis aufgewühlt ...“

(40b) schwere NP [Saltniet 220]

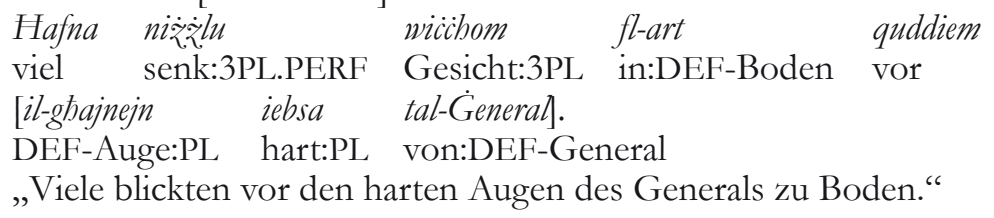


Die Körperteilbezeichnung gநajnejn „Augen“ steht ohne Attribute in der adnominalen Possession wie erwartet im status constructus (40a). Mit dem Attribut iebsa „harte“ in (40b) erfordert dasselbe Possessum aber die Verwendung der für alienable Possession vorgesehenen Konstruktion (mit dem Possessor als Komplement einer $t a^{\prime}$-PP).

Dass es sich bei dieser auf syntaktischer Komplexität beruhenden Regelung um kein Spezifikum des Maltesischen handelt, zeigen u.a. die in (41) aufgeführten Fälle aus dem Albanischen (41c) und Italienischen (41b) im Vergleich zum deutschen Übersetzungsäquivalent (41a).

(41) Schwere Possessum-NPn

(41a) [HP Deutsch 331] Mein Bruder, müsst ihr wissen! [Mein jüngster Bruder]!

(41b) [HP Italienisch 290] Ė mio fratello, sapete? [Il mio fratello più piccolo]!

(41c) [HP Albanisch 252] E dini? Është [vëllai im i vogël]!

Im italienischen Fall (41b) fehlt der definite Artikel, solange die Possessum-NP eine singularische Verwandtschaftsbezeichnung ohne Attribute ist. Schon die Hinzufügung des Adjektivs piccolo „klein“ macht die Possessum-NP relativ schwer und löst dadurch obligatorisch die Setzung des definiten Artikels aus. Ganz ähnlich sollte das Possessum im Albanischen mit dem pränominalen Possessivum auftreten, da es sich um eine „alte“ Verwandtschaftsbezeichnung im Singular handelt. Das Attribut i vogël „kleiner“ hat aber dieselben Auswirkungen wie die Attribuierung im Italienischen, d.h. dass die für alienable Possessa vorgesehene Konstruktion gewählt werden muss: leicht im vëllai „mein Bruder“ mit dem Possessivum links vom Nomen, aber schwer vëllai im i vogël „,mein kleiner Bruder" mit dem Possessivum rechts vom Nomen. Genauso verhält es sich im Georgischen, das bei schweren Possessum-NPn die Suffigierung des Possessivums nicht zulässt (Tamar Reseck persönliche Mitteilung): čemi lamaz̨i deida „meine schöne Tante".

Weder Färöisch (Stolz/Gorsemann 2001, S. 585) noch Isländisch (Stolz et al. 2008, S. 133-134) entziehen sich diesem Mechanismus gänzlich, auch wenn die Details noch zu bestimmen bleiben. Das isländische Beispiel (42) belegt die pränominale Position des Possessivpronomens unter Quantifikation eines Possessums, das einen Körperteil bezeichnet.

$$
\begin{array}{llll}
\text { Isländisch [HP Isländisch 190] } & & \\
\text { Hermione krossađi } & \text { [alla } & \text { sina } & \text { fingur }] . . . \\
\text { Hermine kreuz:PRÄT:3SG all:AKK } & \text { sein:AKK.PL } & \text { Finger } \\
\text { „Hermine hielt alle ihre Finger gekreuzt ... } & &
\end{array}
$$

Unter Verzicht auf die Berücksichtigung von Feinheiten biete ich in (43) die Synopse dieses Abschnitts. Dabei bezeichnet X die volle Wirksamkeit eines Störfaktors. Die Klammerung deutet an, dass das genaue Ausmaß der Störwirkung noch nicht zufriedenstellend eruiert werden konnte. 
(43) Synopse: Störfaktoren

\begin{tabular}{|c|c|c|c|}
\hline Sprache & $\begin{array}{c}\text { Syntaktisches } \\
\text { Gewicht }\end{array}$ & Pragmatik & Semantik \\
\hline Maltesisch & $\mathrm{X}$ & $\mathrm{X}$ & $\mathrm{X}$ \\
\hline Isländisch & $\mathrm{X}$ & $\mathrm{X}$ & $(\mathrm{X})$ \\
\hline Färöisch & $\mathrm{X}$ & $\mathrm{X}$ & $(\mathrm{X})$ \\
\hline Albanisch & $\mathrm{X}$ & \\
\hline Georgisch & $\mathrm{X}$ & & \\
\hline Italienisch & $\mathrm{X}$ & & \\
\hline
\end{tabular}

\section{4. $\quad$ Deutung}

Die obige Darstellung verdeutlicht, dass die Ausprägung des Possessionssystems des Deutschen auch in Europa nicht konkurrenzlos ist. Wo das Deutsche eher nivellierend mögliche possessivische Distinktionen morphosyntaktisch gleich behandelt, nehmen andere Sprachen Europas Unterscheidungen in Form von GP vor, wobei zu einem beträchtlichen Teil ähnliche Prinzipien wirken. Dies ist umso bemerkenswerter als vergleichbare GP-Phänomene in Sprachen verschiedener genetischer Affiliation auftreten (Südkaukasisch, Afroasiatisch, verschiedene indogermanische Sprachen). Selbst in relativ nahen Verwandten des Deutschen wie im Isländischen und Färöischen ist die Alienabilitätskorrelation grammatisch wirksam, und es treten darüber hinaus noch weitere GP-Phänomene auf. Dass innerhalb eines Phylums (Germanisch) solche strukturell auffälligen Unterschiede gegeben sind, lässt ganz besonders aufhorchen. Diese „Mikrovariation“ macht den Untersuchungsgegenstand Possession noch zusätzlich interessant.

Zusammenfassend lässt sich für meine diesmaligen Samplesprachen ein Gesamtbild der GP zeichnen, das auf einem Netzwerk von Faktoren beruht, deren Interaktion komplex anmutet, aber nach recht klaren Regeln funktioniert. Die grammatische Relevanz der GP wird dadurch vielfach opak, dass mit der Pragmatik und dem syntaktischen Gewicht zwei hierarchisch übergeordnete Kräfte am Werk sind, die genug Stärke besitzen, um semantische Distinktionen zu nivellieren. Die Neutralisierungsprozesse sind dabei grundsätzlich so geartet, dass sie die possessivischen Konstruktionen begünstigen, die ansonsten alienable Possession ausdrücken. Das bedeutet jedoch nicht, dass die GP in den Sprachen Europas in grammatischer Hinsicht irre- 
levant ist. Sie wirkt sich morphosyntaktisch überall dort aus, wo die oben identifizierten pragmatischen Bedingungen, das syntaktische Gewicht und/ oder die Differenzierung von Lesarten eines Lexems keinen Einfluss ausüben, also in gewissermaßen „unmarkierten“ Kontexten. Dass diese Kontexte eine eingeschränkte Textfrequenz aufweisen, hebt ja keineswegs die Regeln auf, die in eben diesen Kontexten gelten.

Typologisch bedeutet dies u.a., dass wenigstens einigen Sprachen Europas die Alienabilitätskorrelation nicht fremd ist. In dieser Hinsicht verhalten sich diese Samplesprachen konform zu den zahlreichen außereuropäischen Sprachen, in denen die Unterscheidung von alienabler und inalienabler Possession ein grammatisches Muss ist. Was die hier besprochenen Fälle von den einschlägigen Sprachen anderer Kontinente absetzt, ist die relativ geringe Durchsetzungskraft der GP gegenüber neutralisierenden Faktoren; in den Sprachen Europas scheinen die Kontexte zu dominieren, in denen GP-relevante Kriterien unterdrückbar sind. In einem gewissen Sinne ist es also durchaus richtig zu sagen, dass die Alienabilitätskorrelation in Europa vergleichsweise weniger wichtig ist als anderswo auf dem Globus. Geringere Wichtigkeit und völlige Absenz sind allerdings zwei ganz verschiedene Sachverhalte.

Trotz der möglicherweise geringeren Wichtigkeit der Alienabilitätskorrelation bilden die europäischen Sprachen einen lohnenswerten geolinguistischen Bereich für die Possessionsforschung. Zum einen bieten gerade die starken Restriktionen, denen GP in Europa unterliegt, einen idealen Ansatzpunkt für die Erforschung der Dynamik von possessivischen Systemen. Die Fragen, von wo aus sich GP in einem Possessionssystem ausbreitet und in welche Nischen sie sich eventuell abdrängen lässt, können durch die genaue Analyse der europäischen Gegebenheiten einer Beantwortung näher gebracht werden. Zum anderen bieten nicht nur die in diesem Beitrag beleuchteten Sprachen für die Possessionsforschung vielversprechendes Neuland, da sie nachdrücklich zeigen, dass man sich den Forschungshorizont nicht durch die Fixierung auf den Prototyp einer possessivischen Situation einengen lassen darf. Die Berücksichtigung der so genannten marginalen Possession ist für ein besseres Verständnis der Bauprinzipien von possessivischen Systemen genauso wichtig wie die Beschäftigung mit GP, die auf Eigenschaften des Possessors und/oder der possessivischen Relation fußt. 


\section{Abkürzungen}

$\begin{array}{ll}\text { AKK }=\text { Akkusativ } & \text { N }=\text { Neutrum } \\ \text { CV }=\text { Charaktervokal } & \text { NEG = Negativ } \\ \text { DAT = Dativ } & \text { NOM = Nominativ } \\ \text { DEF = Definit } & \text { PERF }=\text { Perfekt(iv) } \\ \text { F = Femininum } & \text { PL = Plural } \\ \text { GEN = Genitiv } & \text { PP = Präpositionalphrase } \\ \text { GP = gespaltene Possession } & \text { PRÄT = Präteritum } \\ \text { HP = Harry Potter } & \text { PRO = Pronomen } \\ \text { IMPERF = Imperfekt(iv) } & \text { PROG = Progressiv } \\ \text { INDEF = Indefinit } & \text { REF = Reflexiv } \\ \text { INF = Infinitiv } & \text { S = Satz } \\ \text { IO = indirektes Objekt } & \text { SG = Singular } \\ \text { M = Maskulinum } & \text { VP = Verbalphrase }\end{array}$

\section{Primärquellen}

a) Übersetzungen von Joanne K. Rowling (1997): Harry Potter and the Philosopher's Stone. London.

Albanisch - Kasoruho, Amik (Übers.) (2002): Harry Potter dhe guri filozofal. Tirana.

Deutsch - Fritz, Klaus (Übers.) (2000): Harry Potter und der Stein der Weisen. Hamburg.

Färöisch - Hoydal, Gunnar (Übers.) (2000): Harry Potter og vitramannasteinurin. Thorshavn.

Georgisch - Antadze, Manana (Übers.) (2002): Hari P'ot'eri da pilosopiuri kve. Tiflis.

Isländisch - Haraldsdóttir, Helga (Übers.) (1999): Harry Potter og viskusteinninn. Reykjavík.

Italienisch - Astrologo, Marina (Übers.) (2000): Harry Potter e la pietra filosofale. Florenz.

Portugiesisch - Fraga, Isabel (Übers.) (2002): Harry Potter e a pedra filosofal. Barcarena.

b) Maltesische Texte

[Ġahan] - Mifsud, Gorg (1992): Ġahan u hrejjef ohra. Il-Marsa.

[Malta] - Ganado, Herbert (1997): Rajt Malta tinbidel. Bd. IV. Blata 1-Bajda.

[Saltniet] - Aquilina, Gużè (1997): Taht tliet saltniet. Valletta.

[Wenzu] - Zammit, George (1976): Id-dinja ta’ Wenzu u Rozi. Valletta. 


\section{Zitierte Literatur}

Borg, Albert/Azzopardi-Alexander, Marie (1997): Maltese. London.

Buchholz, Oda/Fiedler, Wilfried (1987): Albanische Grammatik. Leipzig.

Butler, Inez M (1988): Gramática zapoteca: Zapoteca de Yatzachi El Bajo. (= Serie gramática de lenguas indígenas de México 4). Mexiko-Stadt.

Chappell, Hilary/McGregor, William (Hg.) (1996): The grammar of inalienability: a typological perspective on body part terms and the part-whole relation. (= Empirical Approaches to Language Typology 14). Berlin u.a.

Corbett, Greville G. (2005): The canonical approach in typology. In: Frajzyngier, Zygmunt/Hodges, Adama/Rood, David S. (Hg.): Linguistic diversity and language theories. (= Studies in Language Companion Series 72). Amsterdam, S. 25-50.

Cunha, Celso/Cintra, Lindley (1985): Nova gramática do português contemporâneo. Lissabon.

Cysouw, Michael/Wälchli, Bernhard (2007): Parallel texts: using translation equivalents in linguistic typology. In: Sprachtypologie und Universalienforschung 60, 2, S. 95-99.

Dahl, Östen/Koptjevskaja-Tamm, Maria (1998): Alienability splits and the grammaticalization of possessive construction. In: Haukoja, Timo (Hg.): Papers from the $16^{\text {th }}$ Scandinavian Conference of Linguistics. Turku, S. 38-49.

Dahl, Östen/Koptjevskaja-Tamm, Maria (2001): Kinship in grammar. In: Baron, Irène/ Herslund, Michael/Sørensen, Finn (Hg.): Dimensions of possession. (= Typological Studies in Language 47). Amsterdam, S. 201-226.

Dixon, Robert M.W. (2010): Basic linguistic theory. 2 Bde. Oxford.

Endruschat, Annette (2007): Durch , mit eingeleitete präpositionale Objekte in den romanischen Sprachen. (= Diversitas Linguarum 13). Bochum.

Evans, Nicholas (2011): Semantic typology. In: Song, Jae Jung (Hg.): The Oxford Handbook of Linguistic Typology. Oxford u.a., S. 504-533.

Fähnrich, Heinz (1986): Kurze Grammatik der georgischen Sprache. Leipzig.

Givón, Talmy (2001): Syntax. An introduction. 2 Bde. 2., überarb. Aufl. Amsterdam u.a.

Haspelmath, Martin (1999): Explaining article-possessor complementarity: economic motivation in noun-phrase syntax. In: Language 75, 2, S. 227-243.

Haspelmath, Martin (2001): The European linguistic area: standard average European. In: Haspelmath, Martin et al. (Hg.): Language Typology and Language Universals. (= Handbücher zur Sprach- und Kommunikationswissenschaft 20). Berlin u.a., S. 1492-1510.

Heine, Bernd (1997): Possession. Cognitive sources, forces, and grammaticalization. (= Cambridge Studies in Linguistics 83). Cambridge u.a.

Hentschel, Gerd/Menzel, Thomas (2002): Zum Ausdruck des unveräußerlichen Besitzes im Russischen: dativische oder präpositionale Markierung des Possessors. In: 
Bublitz, Wolfram/Roncador, Manfred von/Vater, Hans (Hg.): Philologie, Typologie und Sprachstruktur. Festschrift für Winfried Boeder zum 65. Geburtstag. Frankfurt a.M. u.a, S. 323-346.

König, Ekkehard/Haspelmath, Martin (1997): Les constructions à possesseurs externes dans les langues de l'Europe. In: Feuillet, Jacques (Hg.): Actance et valence dans les langues de l'Europe. Berlin u.a., S. 525-606.

Koptjevskaja-Tamm, Maria (1996): Possessive noun phrases in Maltese: alienability, iconicity and grammaticalization. In: Rivista di Linguistica 8, 1, S. 245-274.

Koptjevskaja-Tamm, Maria (2002): Adnominal possession in the European languages: form and function. In: Sprachtypologie und Universalienforschung 55, 2, S. 141172.

Manzelli, Gianguido (2007): Inalienability and emphatic pronominal possession in European and Mediterranean languages: morphosytactic strategies and historical change. In: Ramat, Paolo/Roma, Elisa (Hg.): Europe and the Mediterranean as linguistic areas. Convergencies from a Historical and Typological Perspective. (= Studies in Language Companion Series 88). Amsterdam u.a., S. 159-182.

Nichols, Johanna (1988): On alienable and inalienable possession. In: Shipley, William (Hg.): In honor of Mary Haas: From the Haas Festival Conference on Native American Linguistics. Berlin u.a., S. 557-609.

Nichols, Johanna (1992): Linguistic diversity in space and time. Chicago u.a.

Renzi, Lorenzo/Salvi, Giampolo/Cardinaletti, Anna (Hg.) (1988): Grande grammatica italiana di consultazione I-III. Bologna.

Seiler, Hansjakob (1973): Zum Problem der sprachlichen Possessivität. In: Folia Linguistica 6, S. 231-250.

Seiler, Hansjakob (1983): Possession as an operational dimension of language. (= Language Universals Series 2). Tübingen.

Spanoghe, Anne-Marie (1995): La syntaxe de l'appartenance inaliénable en français, en espagnol et en portugais. (= Studia Romanica et Linguistica 27). Frankfurt a.M. u.a.

Stassen, Leon (2009): Predicative possession. Oxford u.a.

Stefanowitsch, Anatol (2006): Konstruktionsgrammatik und Korpuslinguistik. In: Fischer, Kerstin/Stefanowitsch, Anatol (Hg.): Konstruktionsgrammatik. Von der Anwendung zur Theorie. (= Stauffenburg Linguistik 40). Tübingen, S. 151-176.

Stolz, Thomas (2001): To be with X is to have X: comitatives, instrumentals, locative and predicative possession. In: Linguistics 39, 2, S. 321-350.

Stolz, Thomas (2004): Possessions in the Far North: a glimpse of the alienability correlation in modern Icelandic. In: Premper, Waldfried (Hg.): Dimensionen und Kontinua. Beiträge zu Hansjakob Seilers Universalienforschung. (= Diversitas Linguarum 4). Bochum, S. 73-96.

Stolz, Thomas (2007): Harry Potter meets Le petit prince - on the usefulness of parallel corpora in crosslinguistic investigations. In: Sprachtypologie und Universalienforschung 60, 2, S. 100-117. 
Stolz, Thomas (i.Dr.): The possessive relative clause in Maltese. In: Caruana, Sandro/ Fabri, Ray/Stolz, Thomas (Hg.): Change and variation in Maltese. (= Studia Typologica 9). Berlin.

Stolz, Thomas et al. (2008): Split possession. An areal-linguistic study of the alienability correlation and related phenomena in the languages of Europe. (= Studies in Language Companion Series 101). Amsterdam u.a.

Stolz, Thomas/Gorsemann, Sabine (2001): Pronominal possession in Faroese and the Parameters of Alienability/Inalienability. In: Studies in Language 25, 3, S. 557-599.

Stolz, Thomas/Stroh, Cornelia/Urdze, Aina (2003): Solidaritäten. In: Lingua Posnaniensis 45, S. 69-92.

Thráinsson, Höskuldur (2010): The syntax of Icelandic. Cambridge u.a.

Thráinsson, Höskuldur et al. (2004): Faroese: a handbook and reference grammar. Tórshavn.

Verhoeven, Elisabeth (2007): Experiential constructions in Yucatec Maya. A typologically based analysis of a functional domain in a Mayan language. (= Studies in Language Companion Series 87). Amsterdam u.a.

Zifonun, Gisela (2003): Dem Vater sein Hut. Der Charme des Substandards und wie wir ihm gerecht werden. In: Deutsche Sprache 31, S. 97-126. 
